Artículo

\title{
Rendimiento y biomasa acumulada en frijol común bajo riego y secano
}

\author{
Celia Selene Romero-Félix ${ }^{1 \S}$ \\ Cándido López-Castañeda ${ }^{1}$ \\ Josué Kohashi-Shibata ${ }^{1}$ \\ Carlos Gustavo Martínez-Rueda² \\ Salvador Miranda-Colín ${ }^{1}$ \\ Víctor Heber Aguilar-Rincón ${ }^{1}$ \\ ${ }^{1}$ Campus Montecillo-Colegio de Postgraduados. Carretera México-Texcoco km 36.5, Montecillo, Texcoco, \\ Estado de México, México. CP. 56230. Tel. 595 9520200, ext. 1587. (cgmartinezr@ uaemex.mx). ${ }^{2}$ Facultad \\ de Ciencias Agrícolas-Universidad Autónoma del Estado de México. El Cerrillo, Piedras Blancas, Toluca, \\ Estado de México, México. CP. 50090. (clc@colpos.mx; jkohashi@colpos.mx; smiranda@colpos.mx; \\ aheber@colpos.mx). \\ ${ }^{\S}$ Autora para correspondencia: celiaromero82@hotmail.com.
}

\section{Resumen}

El frijol común (Phaseolus vulgaris L.) cultivado bajo condiciones de secano, se ve afectado por la sequía terminal, reduciendo de manera considerable el rendimiento de semilla y la biomasa acumulada en el dosel de la planta. El objetivo de este estudio fue evaluar la variabilidad en rendimiento de semilla, fenología y biomasa aérea acumulada durante la etapa reproductiva, en un grupo de variedades de frijol común, en condiciones de riego (R) y sequía (S), en el Colegio de Postgraduados, Campus Montecillo. Se utilizaron ocho genotipos de frijol tipo 'Flor de Mayo', tres con testa de color negro y uno nativo, bajo un diseño de bloques completos al azar con tres repeticiones en R y tres en S, en el ciclo verano-otoño 2014. Se utilizó la dosis de fertilización 8040-00. La S disminuyó el rendimiento de semilla (30\%), la biomasa acumulada a inicio de floración (BMIF) (22\%), biomasa acumulada en antesis (BMA) (19\%) y la biomasa acumulada en madurez fisiológica (BMMF) (19\%) y el índice de cosecha (14\%) con respecto a R. La S también redujo los días y grados día a inicio de floración, antesis y madurez fisiológica. Hubo variabilidad dentro del grupo de variedades de frijol evaluadas, resaltando FM 2000 por presentar alto rendimiento de semilla, en promedio de R y S, y en cada condición de humedad (R y S). Mientras que FM RMC sobresalió por exhibir mayor BMIF, BMA y BMMF, tanto en R como en S.

Palabras clave: Phaseolus vulgaris L. fenología, grados día, secano.

Recibido: agosto de 2021

Aceptado: octubre de 2021 


\section{Introducción}

El frijol común (Phaseolus vulgaris L.) es la leguminosa más importante para el consumo humano (Calero et al., 2018). A nivel mundial se estima que más de $60 \%$ de la superficie cultivada se ve afectada por escasez de humedad (Rao et al., 2013). El estrés por sequía ya sea como fenómeno estacional recurrente o como efecto del cambio climático, es actualmente la principal amenaza para el suministro mundial de alimentos (Budak et al., 2013). Este estrés ambiental se convirtió en las últimas décadas un gran desafío para el sustento de los productores de frijol en ambientes marginales o poco desfavorables (Beebe et al., 2013; Asfaw y Blair, 2014), sobre todo en condiciones de secano donde depende de las lluvias que ocurren durante su ciclo de cultivo, por lo que en años donde llueve poco puede reducirse severamente la cantidad y calidad de su producción (Rainey y Griffiths, 2005; Ligarreto et al., 2015); la sequía afecta grandemente al rendimiento de frijol en secano, sobre todo cuando coincide con la etapa reproductiva (Rosales et al., 2012; Osuna et al., 2013); por ejemplo, en variedades de frijol de hábito indeterminado tipo III, el déficit hídrico durante la fase vegetativa, redujo 39\% el rendimiento de semilla, y cuando la sequía se presentó en la floración y el periodo de formación de la semilla, el rendimiento disminuyo 51\% (Acosta y Kohashi, 1989).

Dependiendo de la intensidad, tipo y duración del estrés por sequía, el rendimiento puede disminuir de 20 a 100\% cuando ocurre justo en la etapa reproductiva (López et al., 2011; Beebe et al., 2013). De igual forma la biomasa aérea puede reducirse $25 \%$ en secano con respecto a riego (Barrios et $a l ., 2010)$. El estrés hídrico también puede alterar el ciclo ontogénico del frijol, acortando el número de días a inicio de floración, antesis y madurez fisiológica, así como la duración del intervalo entre la antesis y la madurez fisiológica (Morales et al., 2015).

En condiciones de sequía, la reducción o aumento en el periodo de llenado de grano puede ser ventajoso, Blum (1998) considera que un periodo más corto para el llenado de grano puede permitir cierta evasión del estrés terminal, mientras que una duración más larga permite mayor utilización de las reservas del tallo para el llenado de grano bajo este tipo de estrés.

No obstante, el rendimiento de frijol podría incrementarse si se identifican características agronómicas y fisiológicas que contribuyan a elevar la producción de materia seca y traslocación de asimilados al grano; para alcanzar mayor producción de biomasa aérea se puede incrementar la tasa de crecimiento del cultivo y la tasa de asimilación neta mediante mayor área foliar que favorezca la intercepción y eficiencia en el uso de la radiación (Blum, 2013).

En frijol una alta biomasa aérea está asociada con mayor producción de fotoasimilados que se translocan a vainas y semillas mejorando el rendimiento de grano (Ramírez y Kelly, 1998; Romero et al., 2015), por lo que la identificación de genotipos con alta producción de biomasa aérea, mayor eficiencia en el uso del agua, mayor asignación de fotoasimilados a la formación y llenado de vainas y grano puede ser una estrategia para obtener variedades con mayor tolerancia a sequía (Hall, 2012; Omae et al., 2012). Tomando en cuenta lo anterior, se realizó el presente trabajo de investigación con el objetivo de evaluar la variabilidad en rendimiento de semilla, fenología y biomasa aérea acumulada durante la etapa reproductiva, en un grupo de variedades de frijol común, en condiciones de riego y secano. 


\section{Materiales y métodos}

\section{Sitio experimental}

Este trabajo se realizó en el Campo Experimental del Colegio de Postgraduados, Montecillo, Texcoco, Estado de México (19 $21^{\prime}$ latitud norte, $98^{\circ} 55^{\prime}$ latitud oeste y $2250 \mathrm{msnm}$ ), en el ciclo verano-otoño 2014. El sitio experimental presenta un clima templado subhúmedo tipo $\mathrm{Cb}$ (wo) (w) (i') g con lluvias en verano, temperatura y precipitación media anual de $15.2{ }^{\circ} \mathrm{C}$ y $637 \mathrm{~mm}$, respectivamente (García, 2004). Suelo de textura arcillosa con pH de 8.2, materia orgánica de $2.1 \%$ (Walkey-Black), conductividad eléctrica de $0.5 \mathrm{dS} \mathrm{m}^{-1}$, contenido de nitrógeno de $0.2 \%$ (MicroKjeldhal), fósforo de $9.5 \mathrm{mg} \mathrm{kg}^{-1}\left(\mathrm{P}_{2} \mathrm{O}_{5}\right.$; Olsen) y $1.7 \mathrm{cmol} \mathrm{kg}^{-1}$ de potasio en promedio de los estratos de 0-20, 20-40, 40-60 y 60-80 cm del perfil del suelo.

\section{Material genético}

El germoplasma utilizado incluyó ocho variedades de frijol del tipo 'Flor de Mayo' (FM Anita, FM Corregidora, FM 2000, FM M38, FM Sol, FM Bajío, FM Noura y FM RMC), obtenidas por el INIFAP para áreas de temporal y suelos con humedad favorable en la región del Altiplano Mexicano (Rosales et al., 2004), tres variedades de frijol negro (Criollo San Andrés, Negro Cotaxtla 91 y Negro Veracruz) colectadas en la región sur del estado de Veracruz (Morales et al., 2015) y Michoacán 128 (similar a las variedades tipo 'Flor de Mayo') colectada en Michoacán, México (Barrios et al., 2010). Todas las variedades son de hábito de crecimiento indeterminado tipo III (CIAT, 1982).

\section{Diseño experimental}

Se utilizó un diseño de bloques completos al azar con tres repeticiones en riego $(\mathrm{R})$ y tres en sequía (S), la unidad experimental constó de cuatro surcos de $5 \mathrm{~m}$ de longitud y $0.8 \mathrm{~m}$ de separación. La siembra se realizó el 11 de junio de 2014 en suelo ligeramente húmedo. Se empleó un tratamiento de fertilización de 80-40-00, con urea como fuente de nitrógeno y superfosfato de calcio triple como fuente de fósforo, se aplicó la mitad del nitrógeno y todo el fósforo durante la siembra y la segunda mitad de nitrógeno a los 49 días después de la siembra (dds). Se manejó una densidad de población aproximada de 148000 plantas ha ${ }^{-1}$. Se realizaron labores de cultivo a los 37 y 49 dds. Se aplicó agua a los dos dds en R y S y después a los 25, 33, 44 y 60 dds sólo en R, posteriormente ambos tratamientos de humedad edáfica quedaron sujetos a la precipitación pluvial hasta la madurez fisiológica.

Se aplicó herbicida Flex ${ }^{\circledR}$ (Fomesafen) para el control de malezas de hoja ancha a los 23 dds y herbicida Fusiflex ${ }^{\circledR}$ (Fluazifop-p-butil) para el control de maleza de hoja angosta a los 34 dds. También, se aplicaron insecticidas Afidox ${ }^{\circledR}$ (Dimetoato) a una dosis de $1 \mathrm{~L} \mathrm{ha}^{-1}$ a los $31 \mathrm{y}$ Nugor $^{\circledR}$ (Dimetoato) a los 91 dds para el control de mosquita blanca (Bemisia tabaci) y conchuela, (Epilachna varivestis). Adicionalmente, se aplicó el fertilizante foliar líquido Nutriplant plus ${ }^{\circledR}$ a los 55, 67 y 78 dds. No se observó la presencia de enfermedades foliares durante el ciclo de cultivo. 


\section{Variables de estudio}

\section{Grados día de crecimiento a inicio de floración (GDIF, $\left.{ }^{\circ} \mathrm{Cd}\right)$}

El inicio de floración se determinó cuando 50\% de las plantas en cada unidad experimental presentaron al menos una flor abierta.

\section{Grados día de crecimiento para alcanzar la antesis (GDA, $\left.{ }^{\circ} \mathrm{Cd}\right)$}

Se determinó cuando 50\% de las plantas de cada unidad experimental presentaron flores abiertas.

\section{Grados día de crecimiento para llegar a madurez fisiológica (GDMF, $\left.{ }^{\circ} \mathrm{Cd}\right)$}

Se registró cuando $90 \%$ de las vainas de las plantas perdieron su pigmentación verde (Acosta $e t$ al., 2009). Los grados día (GD, $\left.{ }^{\circ} \mathrm{Cd}\right)$ a inicio de floración, antesis y madurez fisiológica se calcularon con la siguiente ecuación: $\mathrm{GD}=\sum_{\mathrm{i}=0}^{\mathrm{n}}(\overline{\mathrm{X}} \mathrm{i}-\mathrm{Tb})$. Donde $\mathrm{GD}=$ grados día $\left({ }^{\circ} \mathrm{Cd}\right), \overline{\mathrm{X}} \mathrm{i}=$ temperatura media diaria y $\mathrm{Tb}=$ temperatura base con valor de $8.2^{\circ} \mathrm{C}$ para frijol (Barrios y López, 2009).

Biomasa aérea acumulada al inicio de floración (BMIF, $\mathrm{g} \mathrm{m}^{-2}$ ), biomasa aérea acumulada en antesis (BMA, $\mathrm{g} \mathrm{m}^{-2}$ ) y biomasa aérea acumulada en madurez fisiológica (BMMF, $\mathrm{g} \mathrm{m}^{-2}$ )

Se determinaron al cosechar las plantas presentes en un área de $0.4 \mathrm{~m}^{2}$ en los surcos laterales de cada unidad experimental. Las plantas fueron colocadas en una estufa $\left(\right.$ Riossa $\left.^{\circledR}\right)$ con aire forzado a una temperatura de $75^{\circ} \mathrm{C}$ por un periodo de $72 \mathrm{~h}$, para posteriormente obtener su peso seco.

\section{Rendimiento de semilla (RS, $\left.\mathrm{g} \mathrm{m}^{-2}\right)$}

Se determinó al pesar las semillas normales y dividir el peso de la semilla entre el área cosechada.

\section{Índice de cosecha (IC, \%)}

Se calculó al dividir el rendimiento de semilla (RS) entre la biomasa aérea final (BMAF) (100) (Kohashi et al., 1980). Los datos de temperatura $\left({ }^{\circ} \mathrm{C}\right)$ máxima y mínima del aire, y precipitación pluvial ( $\mathrm{mm}$ ) durante el experimento, se registraron diariamente durante el ciclo de las plantas con un termómetro de columna de mercurio de máxima y mínima (marca Taylor), y un pluviómetro portátil, colocados en el lugar donde se llevó a cabo el experimento.

\section{Análisis estadístico}

El análisis de varianza se realizó con el programa estadístico SAS (2009), versión 9.1 para Windows en forma combinada $\mathrm{R}$ y $\mathrm{S}$ como serie de experimentos $\left(\mathrm{Y}_{\mathrm{ijk}}=\mu+\mathrm{G}_{\mathrm{i}}+\mathrm{H}_{\mathrm{j}}+\mathrm{GH}_{\mathrm{ij}}+\mathrm{B}\left(\mathrm{i}_{\mathrm{i}}\right)_{\mathrm{j}}\right.$ $\left.+\mathrm{E}_{\mathrm{ijk}}\right)$, para determinar las diferencias entre los tratamientos de humedad del suelo $(\mathrm{H})$, genotipos (G) y la interacción $\mathrm{G}$ x H. para la comparación de medias se utilizó la prueba de diferencia mínima significativa (DMS, $p \leq 0.05$ ). 


\section{Resultados y discusión}

\section{Datos meteorológicos}

Se observó amplia variación en la temperatura $\left({ }^{\circ} \mathrm{C}\right)$ máxima y mínima del aire, dominando alta temperatura durante la fase reproductiva del cultivo, la cual presentó un valor promedio semanal por arriba de los $25^{\circ} \mathrm{C}$ y temperatura mínima variable con valores medios $>10$ y $7{ }^{\circ} \mathrm{C}$ después de la etapa de antesis (Figura 1).

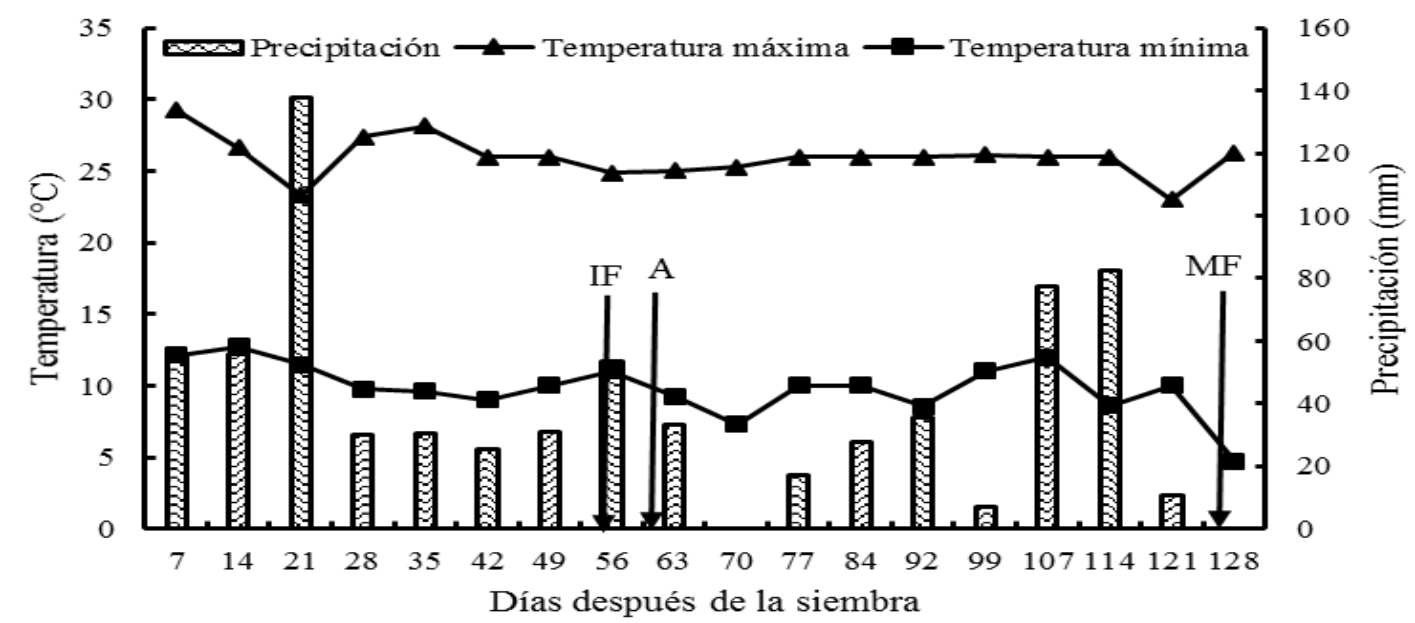

Figura 1. Temperatura máxima y mínima del aire promedio semanal, y precipitación acumulada semanalmente durante el ciclo verano-otoño 2014. Montecillo, Texcoco, Estado de México. IF = inicio de floración; $A=$ antesis; $M F=$ madurez fisiológica.

La precipitación semanal acumulada durante el ciclo de cultivo $(712 \mathrm{~mm})$ también presentó fuertes variaciones, siendo escaza durante la fase reproductiva del cultivo (Figura 1), donde el contenido hídrico de la capa superficial del suelo $(30 \mathrm{~cm})$ disminuyó, alcanzando niveles cercanos al PMP durante el periodo de formación de la semilla, periodo considerado como el más sensitivo a la sequía (Acosta y Kohashi, 1989; Nielsen y Nelson, 1998) y donde el rendimiento (Ambachew et al., 2015; Polania et al., 2016) y calidad de semilla (Rainey y Griffiths, 2005) son más afectados por el estrés hídrico.

\section{Contenido de humedad en el suelo}

Se determinó el contenido de humedad aprovechable (HA) en diferentes estratos del suelo (0-20, 20-40, 40-60 y de 60-80) semanalmente, mediante el método gravimétrico [\% HA= ((peso del suelo húmedo - peso del suelo seco)/peso del suelo seco)) 100]. El contenido de humedad del suelo en $\mathrm{R}$ se mantuvo cerca de capacidad de campo (CC) durante el experimento (datos no mostrados). En condiciones de secano la disponibilidad de humedad del suelo disminuyó conforme avanzó el ciclo del cultivo en los estratos de 0-80 cm, a partir del inicio de floración el nivel de humedad del suelo disminuyó en los estratos que contemplan los 0-60 cm del perfil del suelo, presentando valores por debajo del PMP (Figura 2). 


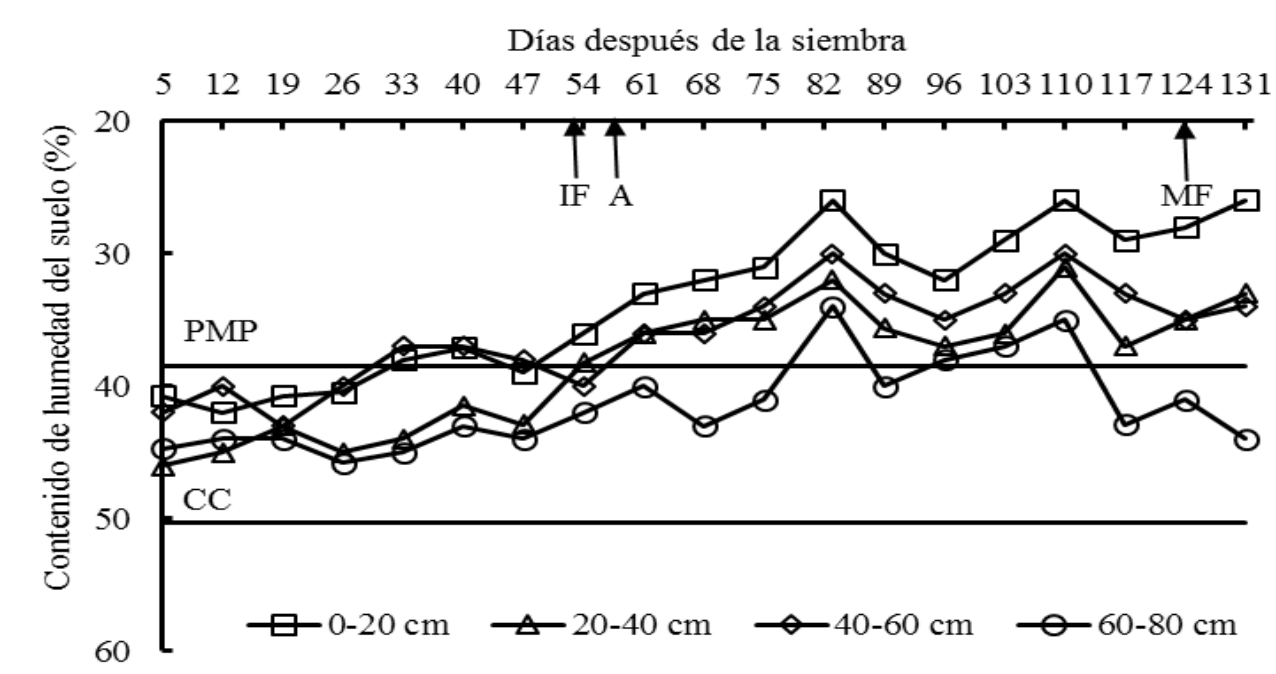

Figura 2. Contenido de humedad del suelo en los estratos de 0-20, 20-40, 40-60 y 60-80 cm en condiciones de secano durante el experimento. Ciclo verano-otoño 2014. Montecillo, Texcoco, Estado de México. $\mathbf{C C}=$ capacidad de campo; $\mathbf{P M P}=$ punto de marchitamiento permanente; $\mathrm{IF}=$ inicio de floración; $\mathrm{A}=$ antesis y $\mathrm{MF}=$ madurez fisiológica.

La capacidad de campo se refiere al estado en el cual un suelo está totalmente mojado después de haber drenado por gravedad, mientras que el porcentaje de marchitez permanente es el contenido hídrico del suelo con el cual las plantas se marchitan (Azcón y Talón, 2013). Chicas et al. (2014), mencionan que la diferencia entre la capacidad de campo y el punto de marchitez permanente determina la capacidad de retención de humedad en los suelos, parámetro con múltiples fines para la planificación agrícola.

\section{Rendimiento de semilla, biomasa acumulada en el dosel vegetal y fenología del cultivo}

El análisis de varianza (Andeva) combinado exhibió diferencias estadísticas significativas entre genotipos para el rendimiento de semilla $(\mathrm{F}=12.06, p<0.0001)$, biomasa aérea acumulada a: inicio de floración $(\mathrm{F}=27.98, p<0.0001)$, antesis $(\mathrm{F}=24.62, p<0.0001)$ y madurez fisiológica $(\mathrm{F}=4.33$, $p<0.0002)$, grados día acumulados: a inicio de floración $((\mathrm{F}=16.03, p<0.0001)$, antesis $(\mathrm{F}=16.63$, $p<0.0001)$ y madurez fisiológica $(\mathrm{F}=10.07, p<0.0001)$. No hubo diferencias estadísticas entre variedades para el índice de cosecha $(\mathrm{F}=1.73, p>0.05)$.

La comparación de medias entre genotipos mostró que FM 2000 tuvo mayor rendimiento de semilla, FM RMC mayor biomasa aérea acumulada a inicio de floración; FM RMC y FM Sol alta biomasa aérea acumulada en antesis, FM 2000, FM RMC, FM Sol, Michoacán 128 y FM Corregidora mayor biomasa aérea acumulada en madurez fisiológica; FM M38, Michoacán 128, FM Noura, FM Corregidora y Negro Cotaxtla 91 alto número de días y grados día a inicio de floración; FM M38, Michoacán 128, FM Noura, FM Corregidora, Criollo San Andrés, Negro Cotaxtla 91 y Negro Veracruz alto número de días o grados día a antesis y FM 2000, FM M38, FM Noura, FM Corregidora y Negro Cotaxtla 91 alto número de días o grados día a madurez fisiológica que las otras variedades en promedio de R y S (Cuadro 1). 
Cuadro 1. Rendimiento de semilla, biomasa aérea y fenología de 12 variedades de frijol en promedio de riego y sequía. Ciclo verano-otoño 2014. Montecillo, Texcoco, Estado de México.

\begin{tabular}{|c|c|c|c|c|c|c|c|c|}
\hline Variedades & $\mathrm{RS}$ & BMIF & BMA & BMMF & IC & GDIF & GDA & GDMF \\
\hline FM 2000 & 238 & 51 & 75 & 411 & 44 & $514(52)$ & $560(56)$ & 1248 (130) \\
\hline FM RMC & 196 & 86 & 105 & 421 & 40 & $502(50)$ & $556(56)$ & $1180(122)$ \\
\hline FM M38 & 196 & 46 & 77 & 274 & 45 & $543(55)$ & $587(59)$ & 1253 (131) \\
\hline FM Sol & 115 & 58 & 105 & 356 & 37 & 485 (49) & $538(54)$ & $1162(120)$ \\
\hline Michoacán 128 & 136 & 53 & 83 & 355 & 36 & $570(57)$ & $607(61)$ & $1207(125)$ \\
\hline FM Noura & 166 & 58 & 87 & 287 & 38 & $564(57)$ & $600(61)$ & $1281(134)$ \\
\hline FM Anita & 137 & 42 & 72 & 236 & 45 & $500(50)$ & $554(56)$ & $1166(121)$ \\
\hline FM Corregidora & 130 & 49 & 90 & 331 & 36 & $551(55)$ & $592(60)$ & 1277 (133) \\
\hline FM Bajío & 162 & 37 & 64 & 206 & 43 & $551(53)$ & $571(58)$ & 1152 (119) \\
\hline Criollo San Andrés & 139 & 37 & 59 & 233 & 40 & $541(54)$ & $584(59)$ & $1204(125)$ \\
\hline Negro Cotaxtla 91 & 114 & 37 & 57 & 167 & 39 & $560(56)$ & $600(61)$ & $1251(130)$ \\
\hline Negro Veracruz & 110 & 37 & 48 & 243 & 37 & $541(54)$ & $584(59)$ & $1222(127)$ \\
\hline Media general & 154 & 49 & 77 & 293 & 40 & $533(53)$ & $578(58)$ & 1217 (126) \\
\hline DMS $(p \leq 0.05)$ & 37 & 8 & 10 & 111 & 7 & $20(2)$ & $15(2)$ & $41(5)$ \\
\hline
\end{tabular}

$\mathrm{RS}=$ rendimiento de semilla $\left(\mathrm{g} \mathrm{m}^{-2}\right) ; \mathrm{BMIF}=$ biomasa aérea acumulada a inicio de floración $\left(\mathrm{g} \mathrm{m}^{-2}\right) ; \mathrm{BMA}=$ biomasa aérea acumulada en antesis $\left(\mathrm{g} \mathrm{m}^{-2}\right)$; BMMF = biomasa aérea acumulada en madurez fisiológica $\left(\mathrm{g} \mathrm{m}^{-2}\right)$; $\mathrm{IC}=$ índice de cosecha (\%); GDIF= grados día acumulados $\left(\mathrm{GD},{ }^{\circ} \mathrm{Cd}\right)$ a inicio de floración (IF); GDA= grados día acumulados (GD, ${ }^{\circ} \mathrm{Cd}$ ) en antesis (A); GDMF= grados día acumulados $\left(\mathrm{GD},{ }^{\circ} \mathrm{Cd}\right)$ en madurez fisiológica $(\mathrm{MF}) ; \mathrm{DMS}=$ valor de la diferencia mínima significativa para la comparación entre genotipos.

El rendimiento de semilla y la biomasa aérea final, en promedio de variedades, obtenidos en este trabajo fueron 34 y $22 \%$ menores comparados con los resultados de Barrios et al. (2010), mientras que el número de días a madurez fisiológica se prolongó 20 días con respecto a lo reportado por Barrios et al. (2010), en variedades de frijol tipo 'Flor de Mayo' en promedio de tres ambientes. El ciclo de cultivo y la temperatura juegan un papel determinante en las etapas fenológicas de las plantas, por lo cual, estas diferencias en parte se atribuyen a que en este estudio la siembra se llevó a cabo de manera tardía en verano-otoño y la temperatura máxima promedio fluctuó entre $25^{\circ} \mathrm{C}$ y Barrios et al. (2010) sembraron en primavera-verano y la temperatura promedio durante el ciclo de cultivo fue de $31.7^{\circ} \mathrm{C}$, acelerando las etapas de inicio de floración y madurez fisiológica.

\section{Riego vs secano}

El Andeva combinado para las dos condiciones de humedad detectó diferencias significativas para el rendimiento de semilla $(\mathrm{F}=70.82, p<0.0001)$, biomasa aérea acumulada: al inicio de floración $(\mathrm{F}=62.71, p<0.0001)$, antesis $(\mathrm{F}=58.5, p<0.0001)$ y en madurez fisiológica $(\mathrm{F}=7.87, p=0.0073)$, índice de cosecha $(\mathrm{F}=16.58, p=0.0002)$, grados día acumulados a: inicio de floración $(\mathrm{F}=24.28$, $p<0.0001)$, antesis $(\mathrm{F}=18.74, p<0.0001)$ y madurez fisiológica $(\mathrm{F}=10.81, p=0.0019)$. 
El estrés por sequía redujo la expresión de todos los caracteres evaluados en este estudio; el rendimiento de semilla, la biomasa aérea final, biomasa aérea acumulada a inicio de floración, biomasa aérea acumulada en antesis, biomasa aérea acumulada durante la madurez fisiológica y el índice de cosecha, en promedio de genotipos, disminuyeron 30, 20, 22, 19, 19 y 14\% en sequía con respecto a riego (Cuadro 2).

Cuadro 2. Rendimiento de semilla, biomasa aérea y fenología en promedio de 12 variedades de frijol en riego y sequía. Ciclo verano-otoño 2014. Montecillo, Texcoco, Estado de México.

\begin{tabular}{ccccccccc}
\hline Nivel de humedad & RS & BMIF & BMA & BMMF & IC & GDIF & GDA & GDMF \\
\hline Riego & 181 & 55 & 85 & 325 & 43 & $543(54)$ & $584(59)$ & $1230(128)$ \\
Sequía & 126 & 43 & 69 & 262 & 37 & $523(52)$ & $571(57)$ & $1199(124)$ \\
DMS $(p \leq 0.05)$ & 13 & 3 & 4 & 45 & 3 & $8(1)$ & $6(1)$ & $19(2)$ \\
\hline
\end{tabular}

$\mathrm{RS}=$ rendimiento de semilla $\left(\mathrm{g} \mathrm{m}^{-2}\right) ; \mathrm{BMIF}=$ biomasa aérea acumulada a inicio de floración $\left(\mathrm{g} \mathrm{m}^{-2}\right) ; \mathrm{BMA}=$ biomasa aérea acumulada en antesis $\left(\mathrm{g} \mathrm{m}^{-2}\right)$; BMMF= biomasa aérea acumulada en madurez fisiológica $\left(\mathrm{g} \mathrm{m}^{-2}\right)$; IC $=$ índice de cosecha (\%); GDIF= grados día acumulados $\left(\mathrm{GD},{ }^{\circ} \mathrm{Cd}\right)$ a inicio de floración (IF); GDA= grados día acumulados (GD, ${ }^{\circ} \mathrm{Cd}$ ) en antesis $(\mathrm{A})$; GDMF $=$ grados día acumulados $\left(\mathrm{GD},{ }^{\circ} \mathrm{Cd}\right)$ en madurez fisiológica $(\mathrm{MF}) ; \mathrm{DMS}=$ valor de la diferencia mínima significativa para la comparación entre genotipos.

La sequía también provocó una reducción en los días después de la siembra y grados día acumulados (GD) al inicio de la floración (20 GD), antesis (13 GD) y madurez fisiológica (31 GD) (Cuadro 2). La tendencia de estos caracteres a disminuir al pasar de riego a sequía en condiciones de secano ha sido observada en otros estudios; el rendimiento de semilla, biomasa aérea, índice de cosecha (Acosta et al., 2004, 2009; Barrios et al., 2010; Romero et al., 2019), días para el inicio de floración (Barrios et al., 2010), días a antesis y días a madurez fisiológica (Tosquy et al., 2017; Romero et al., 2018).

La biomasa acumulada en las etapas de inicio de floración, antesis y madurez fisiológica en todas las variedades fue menor en sequía que en riego, ya que en sequía los genotipos presentaron menor número de días para alcanzar cada una de estas etapas fenológicas, reduciendo el periodo para la acumulación de la biomasa y la efectiva movilización de asimilados a la formación y crecimiento del grano; la sequía influye en la duración de las etapas fenológicas (Rosales et al., 2001); por su parte, Asfaw et al. (2012) declara que la senescencia de las hojas puede reducir la duración del período fotosintético, así como la tasa de fotosíntesis; sin embargo, la senescencia de la hoja también puede contribuir de manera efectiva al período de llenado del grano a través de la removilización de carbono desde los tejidos vegetativos hacia el grano.

En riego, se detectaron diferencias estadísticas significativas entre variedades para el rendimiento de semilla $(\mathrm{F}=6.95, p<0.0001)$, biomasa aérea acumulada a: inicio de floración $(\mathrm{F}=26.71, p<$ $0.0001)$, antesis $(\mathrm{F}=12.33, p<0.0001)$ y madurez fisiológica $(\mathrm{F}=2.19, p=0.05)$, grados día acumulados a: inicio de floración $(\mathrm{F}=8.47, p<0.0001)$, antesis $(\mathrm{F}=6, p<0.0002)$ y madurez fisiológica $(\mathrm{F}=3.57, p=0.0054)$. No se observaron diferencias estadísticas significativas para el índice de cosecha $(\mathrm{F}=1.29, p>0.05)$. 
Las variedades FM 2000, FM M38 y FM RMC tuvieron mayor rendimiento de semilla (Figura 3a); FM RMC mostró alta acumulación de biomasa a inicio de floración (Figura 3b); FM RMC y FM Sol mayor acumulación de biomasa al llegar a antesis (Figura 3c) y FM 2000, FM RMC, FM Sol, Michoacán 128, FM Corregidora y FM Noura (Figura 3d) tuvieron mayor acumulación de biomasa aérea en madurez fisiológica que las otras. Por otro lado, FM M38, FM Noura, Michoacán 128, FM Corregidora y Negro Cotaxtla 91 mostraron mayor acumulación de grados día o días a inicio de floración (Figura 3f), antesis (Figura 3g) y madurez fisiológica (Figura 3h). También, Negro Veracruz tuvo mayor acumulación de grados día a madurez fisiológica (Figura 3h).
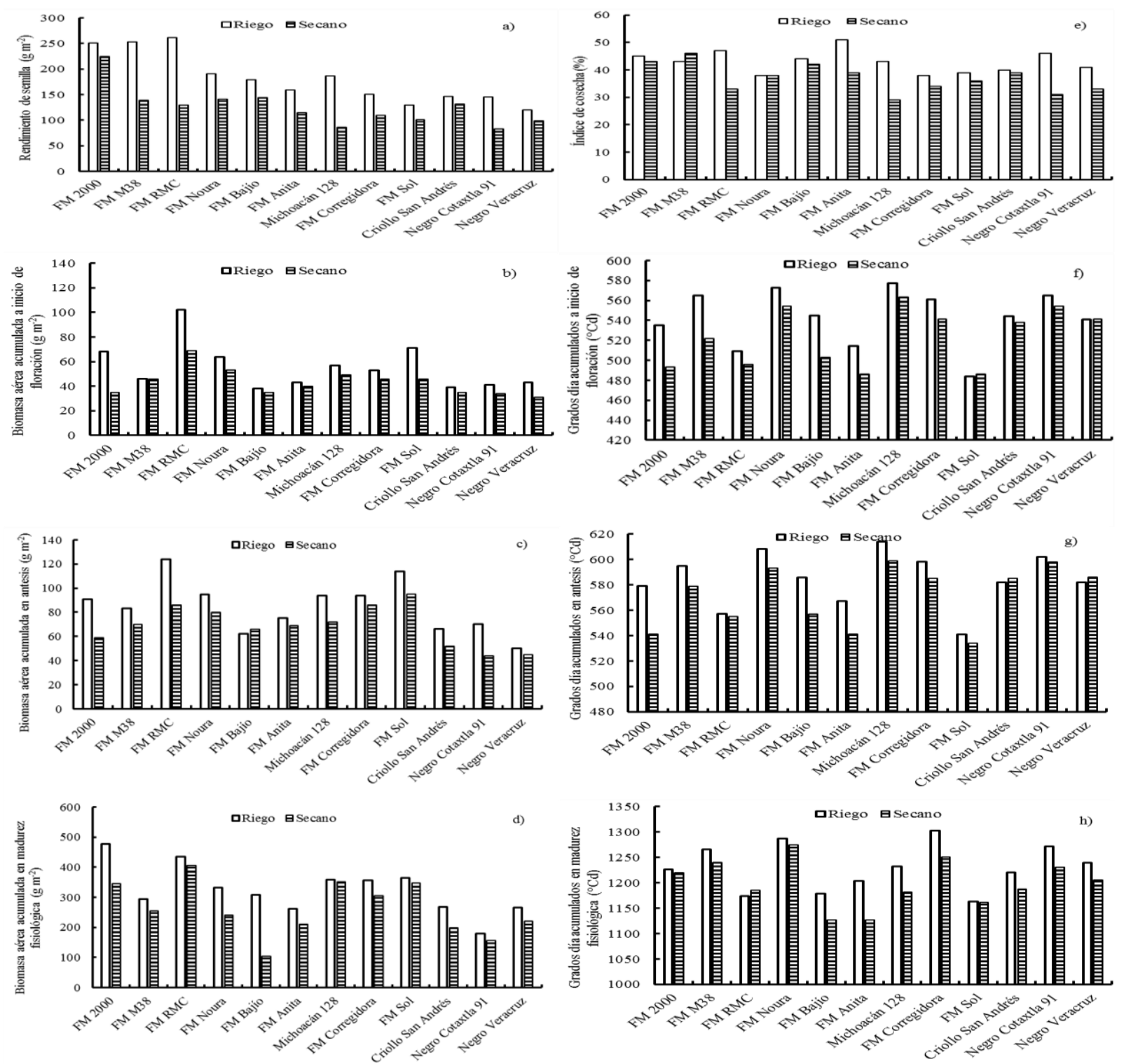

Figura 3. Rendimiento de semilla (a); biomasa aérea acumulada a inicio de floración (b), en antesis (c) y en madurez fisiológica (d); índice de cosecha (e); grados día acumulados a inicio de floración (f), en antesis (g) y en madurez fisiológica; y (h) de 12 variedades de frijol bajo riego y secano. Ciclo verano-otoño 2014. Montecillo, Texcoco, Estado de México. 
En condiciones de sequía, se observaron diferencias estadísticas significativas para todos los caracteres evaluados; rendimiento de semilla $(\mathrm{F}=10.9, p<0.0001)$, biomasa aérea acumulada a: inicio de floración $(\mathrm{F}=7.58, p<0.0001)$, antesis $(\mathrm{F}=15.39, p<0.0001)$ y madurez fisiológica $(\mathrm{F}=$ 2.86, $p=0.01)$, índice de cosecha $(\mathrm{F}=2.53, p=0.03)$, grados día acumulados: a inicio de floración $(\mathrm{F}=8.66, p<0.0001)$, antesis $(\mathrm{F}=13.62, p<0.0001)$ y madurez fisiológica $(\mathrm{F}=4.16, p=0.0022)$.

Las variedades tuvieron un comportamiento diferencial en el rendimiento de semilla, fenología y biomasa aérea acumulada en cada una de sus etapas fenológicas en sequía; la variedad FM 2000 presentó mayor rendimiento de semilla (Figura 3a); FM RMC mayor biomasa aérea acumulada a inicio de floración (Figura 3b); FM RMC, FM Corregidora y FM Sol mayor biomasa aérea acumulada en antesis (Figura 3c); FM 2000, FM M38, FM RMC, Michoacán 128, FM Corregidora y FM Sol mayor biomasa aérea acumulada en madurez fisiológica (Figura 3d) y FM 2000, FM M38, FM Noura, FM Bajío, FM Anita y Criollo San Andrés mayor índice de cosecha (Figura 3e). Mientras que las variedades FM Noura, Michoacán 128, FM Corregidora, Criollo San Andrés, Negro Cotaxtla 91 y Negro Veracruz acumularon mayor cantidad en grados día para el inicio de floración (Figura 3f) y antesis (Figura 3g) las variedades FM 2000, FM M38, FM Noura, FM Corregidora y Negro Cotaxtla 91 mostraron mayor acumulación de grados día durante la madurez fisiológica (Figura 3h).

En la interacción genotipos por niveles de humedad, los genotipos FM RMC, FM M38, Michoacán 128 y Negro Cotaxtla 91; FM 2000, FM RMC y FM Sol; y FM 2000, FM RMC, Michoacán 128 y Negro Cotaxtla 91 mostraron mayores reducciones en rendimiento de semilla, biomasa aérea acumulada a inicio de floración y biomasa aérea acumulada en antesis al pasar de riego a sequía. Las variedades que presentan mayor disminución en el rendimiento o algún otro componente del rendimiento o característica al pasar de riego a sequía, se consideran variedades susceptibles a sequía (Romero et al., 2019).

La relación entre el rendimiento de semilla en riego y sequía indicó que la variedad FM 2000 fue más tolerante a sequía y también tuvo buen comportamiento en riego; similarmente, Polania et al. (2016) identificaron genotipos de frijol común que además de mostrar tolerancia a sequía, tuvieron alto rendimiento en condiciones favorables de humedad, donde las líneas resistentes a sequía debieron su mejor comportamiento a un alto uso efectivo del agua e índice de cosecha.

En condiciones de riego las variedades de frijol tipo 'Flor de Mayo' y con semillas de testa negra, tuvieron los más altos rendimiento de semilla que aquellas que estuvieron bajo condiciones de secano; el rendimiento de semilla (RS) se relacionó positiva y significativamente con la biomasa aérea acumulada a inicio de floración $(\mathrm{BMIF})$ en riego $[\mathrm{RS}=1.42(\mathrm{BMIF})+102.2, \mathrm{r}=0.53, p \leq$ 0.05] (Figura 4a), mientras que en secano $[\mathrm{RS}=-0.25(\mathrm{BMIF})+135.9]$ la relación fue positiva $\mathrm{y}$ no significativa ( $p>0.05$ ) (Figura 4a) en riego, el rendimiento de semilla se relacionó de forma positiva y significativa con la biomasa aérea acumulada en la madurez fisiológica (BMMF) $[\mathrm{RS}=$ $0.37(\mathrm{BMMF})+60.5, \mathrm{r}=0.61, p \leq 0.05]$ (Figura $4 \mathrm{~b}$ ) y en secano, la relación fue positiva pero no significativa $(p>0.05)$ (Figura $4 b)$ el rendimiento de semilla se relacionó de forma positiva pero no significativa con el índice de cosecha (IC) en riego $[\mathrm{RS}=4.09$ (IC) $+4.96, \mathrm{r}=0.33, p>0.05]$ (Figura 4c); sin embargo, en secano la relación fue positiva y significativa $[\mathrm{RS}=5.29$ (IC) -70.18 , $\mathrm{r}=0.72, p \leq 0.005]$ (Figura 4c). 

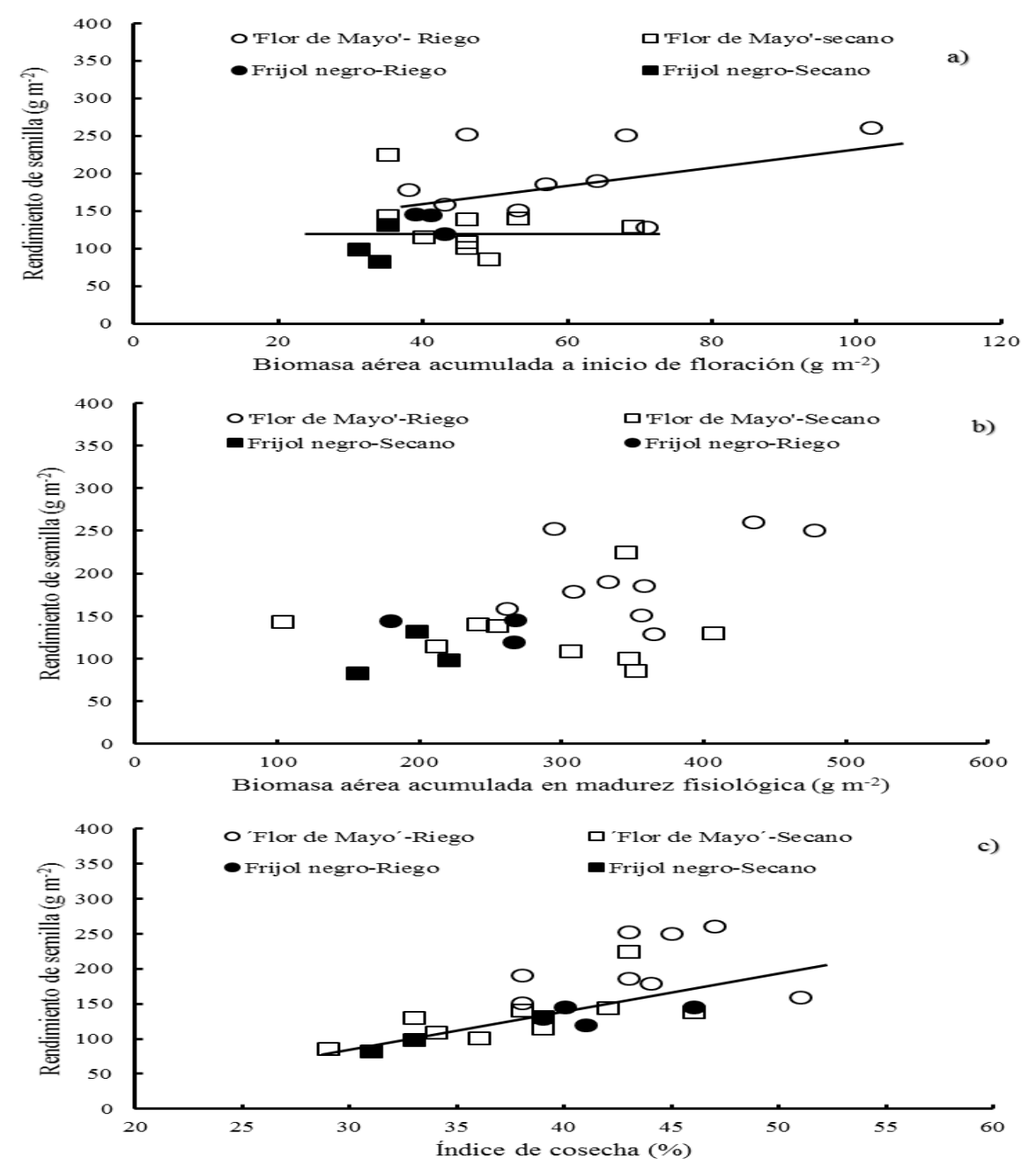

Figura 4. Relación entre el rendimiento de semilla con la biomasa aérea final (a) y el índice de cosecha (b) en riego y sequía para las variedades de frijol tipo 'Flor de Mayo' y semillas con testa de color negro. Ciclo verano-otoño 2014. Montecillo, Texcoco, Estado de México.

En otro trabajo de investigación, se observó una correlación positiva y significativa entre el rendimiento de semilla y el índice de cosecha en promedio de 25 variedades de frijol común en condiciones de sequía en Ethiopia (Asfaw y Blair, 2014) y una correlación positiva y significativa entre el rendimiento de semilla y la biomasa aérea en promedio de cinco variedades de frijol común de diferente hábito de crecimiento en riego y sequía (Ramírez y Kelly, 1998).

\section{Conclusiones}

Se observó variabilidad dentro del grupo de variedades de frijol evaluadas, resaltando FM 2000 por presentar alto rendimiento de semilla, en promedio de riego y sequía y en cada condición de humedad (riego y sequía). Mientras que la variedad FM RMC sobresalió por exhibir mayor biomasa aérea acumula a inicio de floración, antesis y madurez fisiológica, tanto en riego como en sequía. La sequía en condiciones de secano disminuyó el rendimiento de semilla y la biomasa aérea acumulada a inicio de floración, antesis y madurez fisiológica, así como el número de días a inicio de floración, antesis y madurez fisiológica. 


\section{Literatura citada}

Acosta, D. E.; Trejo, L. C.; Ruiz, P. L. del Mar.; Padilla, R. J. C. y Acosta, G. J. A. 2004. Adaptación del frijol a sequía en la etapa reproductiva. Terra Latinoam. 22(1):49-58. http://redalyc.uaemex.mx/src/inicio/ArtPdfRed.jsp?iCve=57311208006.

Acosta, D. E.; Acosta, G. J. A.; Trejo, L. C.; Padilla, R. J. S. and Amador, R. M. D. 2009. Adaptation traits in dry bean cultivars grown under drought stress. Agric. Téc. Méx. 35(4):416-425.

Acosta, G. J. A and Kohashi, S. J. 1989. Effect of water stress on growth and yield of indeterminate dry-bean (Phaseolus vulgaris) cultivars. Field Crops Res. 20(2):81-93. https://doi.org/10. 1016/0378-4290(89)90054-3.

Ambachew, D.; Mekbib, F.; Asfaw, A.; Beebe, S. E. and Blair, M. W. 2015. Trait associations in common bean genotypes grown under drought stress and field infestation by BSM bean fly. The Crop J. 3(4):305-316. https://doi.org/10.1016/j.cj.2015.01.006.

Azcón, B. J. y Talon, M. 2013. Fundamentos de fisiología vegetal. 2a . (Ed.). en español. McGrawHill Interamericana de España, S. L. 651 p.

Asfaw, A.; Blair, M. W. and Struik, P. C. 2012. Multienvironment quantitative trait loci analysis for photosynthate acquisition, accumulation, and remobilization traits in common bean under drought stress. G3: Genes, Genomes, Genetics. 2(5):579-595. doi: 10.1534/g3. 112.002303.

Asfaw, A. and Blair, M. W. 2014. Quantification of drought tolerance in ethiopian common bean varieties. Agric. Sci. 5(2):124-139. http://dx.doi.org/10.4236/as.2014.52016.

Barrios, G. E. J. y C. López-Castañeda. 2009. Temperatura base y tasa de extensión foliar en frijol. Agrociencia. 43(1):25-35.

Barrios, G. E. J.; López, C. C.; Kohashi, S. J.; Acosta, G. J. A.; Miranda, C. S. y Mayek, P. N. 2010. Rendimiento de semilla y sus componentes en frijol flor de mayo en el centro de México. Agrociencia. 44(4):481-489.

Beebe, S. E.; Rao, I. M.; Blair, M. W. and Acosta-Gallegos, J. A. 2013. Phenotyping common beans for adaptation to drought. Frontiers in Physiol. 4(35):1-20. doi: 10.3389/fphys. 2013.00035.

Blum, A. 1998. Improving wheat grain filling under stress by stem reserve mobilisation. Euphytica. 100(1-3):77-83. https://doi.org/10.1023/A:1018303922482.

Blum, A. 2013. Heterosis, stress, and the environment: a possible road map towards the general improvement of crop yield. J. Exp. Bot. 64(16):4829-4837. doi:10.1093/jxb/ert289.

Budak, H.; Kantar, M. and Kurtoglu, K. Y. 2013. Drought tolerance in modern and wild wheat. Sci. World J. 2013(548246):1-16. http://dx.doi.org/10.1155/2013/548246.

Chicas, S. R. A.; Vanegas, C. E. A. y García, A. N. 2014. Determinación indirecta de la capacidad de retención de humedad en suelos de la subcuenca del río Torjá, Chiquimula, Guatemala. Rev. Cienc. Técnic. Agropec. 23(1):41-46. http://scielo.sld.cu/pdf/rcta/v23n1/rcta 07114.pdf.

Calero, A.; Castillo, Y.; Quintero, E.; Pérez, Y. y Olivera, D. 2018. Efecto de cuatro densidades de siembra en el rendimiento agrícola del frijol común (Phaseolus vulgaris L.). Rev. Fac. Cienc. 7(1):88-100. https://doi.org/10.15446/rev.fac.cienc.v7n1.67773.

CIAT. 1982. Centro Internacional de Agricultura Tropical. Etapas de desarrollo de la planta de frijol común. Cali, Colombia. 26 p. 
García, E. 2004. Modificaciones al sistema de clasificación climática de Köppen (para adaptarlo a las condiciones de la República Mexicana $5^{\text {ta. }}$ (Ed.). Instituto de GeografíaUniversidad Naional Autónoma de México (UNAM) (CD con el programa modifica). México, DF. 71 p.

Hall, A. E. 2012. Phenotyping cowpeas for adaptation to drought. Frontiers in physiology. 3(155):1-8. Doi: 10.3389/fphys.2012.00155.

Kohashi, S. J.; da Costa, J. C. and Miranda, S. C. 1980. Harvest index in Phaseolus vulgaris (L.). Ann. Rep. Bean Improv. Coop. 23:87-89. https://www.alice.cnptia.embrapa.br/bitstream/ doc/885721/1/BIC198001.pdf.

Ligarreto, M. G. A.; Castro, H. O. A. and Cháves, B. 2015. Estabilidad fenotípica de una colección de fríjol andino (Phaseolus vulgaris L.) tipo arbustivo. Rev. UDCA Actualidad and Divulgación Científica. 18(1):109-118. https://revistas.udca.edu.co/index.php/ruadc/ article/view/459.

López, S. E.; Acosta, G. J. A.; Tosquy, V. O. H.; Salinas, Pérez, R. A.; Sánchez, G. B. M.; Rosales, S. R.; González, R. C.; Moreno, G. T.; Villar, S. B.; Cortinas, E. H. M. y Zandate, H. R. 2011. Estabilidad de rendimiento en genotipos mesoamericanos de frijol de grano negro en México. Rev. Mex. Cienc. Agríc. 2(1):29-40.

Morales, R. A.; López, C. C.; Kohashi, S. J.; Miranda, C. S. y García, E. A. 2015. Comparación de los componentes del rendimiento en variedades de frijol en condiciones de acidez y humedad residual del suelo en el sur de Veracruz. Terra Latinoam. 33(4):309-319.

Nielsen, D. C. and Nelson, N. O. 1998. Black bean sensitivity to water stress at various growth stages. Crop Sci. 38(2):422-427. https://doi.org/10.2135/cropsci1998.0011183X00380002 $0025 \mathrm{x}$.

Omae, H.; Kumar, A. and Shono, M. 2012. Adaptation to high temperature and water deficit in the common bean (Phaseolus vulgaris L.) during the reproductive period. J. Bot. 2012(803413):1-6. doi:10.1155/2012/803413.

Osuna, C. E. S.; Reyes, M. L.; Padilla, R. J. S.; Rosales, S. R.; Martínez, G. M. A.; Acosta, G. J. A. y Figueroa, S. B. 2013. Rendimiento de genotipos de frijol con diferentes métodos de siembra y riego-sequía en Aguascalientes. Rev. Mex. Cienc. Agríc. 4(8):1209-1221.

Polania, J.; Poschenrieder, C.; Rao, I. and Beebe, S. 2016. Estimation of phenotypic variability in symbiotic nitrogen fixation ability of common bean under drought stress using $15 \mathrm{~N}$ natural abundance in grain. European J. Agron. 79:66-73. http://dx.doi.org/10.1016/j.eja.2016. 05.014 .

Rainey, K. M. and Griffiths, P. D. 2005. Differential response of common bean genotypes to high temperature. J. Am. Soc. Hortic. Sci. 130(1):18-23. https://doi.org/10.21273/JASHS.130. 1.18 .

Ramirez, V. P. and Kelly, J. D. 1998. Traits related to drought resistance in common bean. Euphytica. 99(2):127-136. https://doi.org/10.1023/A:1018353200015.

Rao, I.; Beebe, S.; Polania, J.; Ricaurpe, J.; Cajiao, C.; Garcia, R. and Rivera, M. 2013. Can Tepary bean be a model for improvement of drought resistance in common bean? Afri. Crop Sci. J. 21(4):265-281.

Romero, F. C. S.; López, C. C.; Miranda, C. S.; Kohashi, S. J.; Aguilar, R. V. H. y Martínez, R. C. G. 2015. Variabilidad del rendimiento de semilla y sus componentes en frijol común bajo condiciones de temporal. Ciencias Agrícolas Informa. 24(1):7-17.

Romero, F. C. S.; López, C. C.; Kohashi, S. J.; Martínez, R. C. G.; Miranda, C. S. y Aguilar, R. V. H. 2018. Ambiente y genotipo: efectos en el rendimiento y sus componentes, y fenología en frijol común. Acta Universitaria. 28(6):20-32. doi: 10.15174/au2018.1760. 
Romero, F. C. S.; López, C. C.; Kohashi, S. J.; Miranda, C. S.; Aguilar, R. V. H. y Martínez, R. C. G. 2019. Cambios en el rendimiento y sus componentes en frijol bajo riego y sequía. Rev. Mex. Cienc. Agríc. 10(2):351-364.

Rosales, M. A.; Ocampo, E.; Rodríguez, V. R.; Olvera, C. Y.; Acosta, G. J. A; Covarrubias, A. A. 2012. Physiological analysis of common bean (Phaseolus vulgaris L.) cultivars uncovers characteristics related to terminal drought resistance. Plant Physiol. Biochem. 56(2012):2434. doi:10.1016/j.plaphy.2012.04.007.

Rosales, S. R.; Ochoa, M. R. y Acosta, G. J. A. 2001. Fenología y rendimiento del frijol en el altiplano de México y su respuesta al fotoperiodo. Agrociencia. 35(5):513-523.

Rosales, S. R.; Acosta, G. J. A.; Muruaga, M. J. S.; Hernández, C. J. M.; Esquivel, E. G. y Pérez, H. P. 2004. Variedades mejoradas de frijol del Instituto Nacional de Investigaciones Forestales, Agrícolas y Pecuarias (INIFAP). Libro técnico 6. 148 p.

SAS. 2009. The SAS System Program release 9.1 for Windows. SAS Institute, Inc. Cary, North Carolina, USA. Software of statistical analysis.

Tosquy, V. O. H.; López, S. E.; Zetina, L. R.; Villar, S. B. y Rodríguez, R. J. R. 2017. Producción de genotipos de frijol negro en condiciones de humedad residual y sequía terminal. Terra Latinoam. 35(1):29-39. 